\title{
OUTCOMES OF ENDOVASCULAR THROMBECTOMY WITH AND \\ WITHOUT BRIDGING THROMBOLYSIS FOR ACUTE LARGE VESSEL \\ OCCLUSION ISCHAEMIC STROKE
}

\begin{abstract}
Authors and Affiliations:
Julian Maingard ${ }^{\mathrm{b}, \mathrm{f}}$, Yasmin Shvarts ${ }^{\mathrm{b}}$, Ronan Motyer ${ }^{\mathrm{a}}$, Vincent Thijs ${ }^{\mathrm{d}}$, Paul Brennan ${ }^{\mathrm{a}}$, Alan O’Hare ${ }^{\mathrm{a}}$, Seamus Looby ${ }^{\mathrm{a}}$, John Thornton ${ }^{\mathrm{a}}$, Joshua A Hirsch ${ }^{\mathrm{i}}$, Christen D

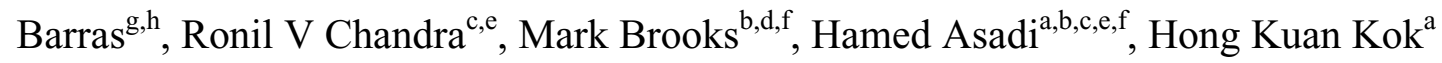

${ }^{\mathrm{a}}$ Interventional Neuroradiology Service, Department of Radiology, Beaumont Hospital, Dublin 9, Ireland.

${ }^{\mathrm{b}}$ Interventional Neuroradiology Service, Radiology Department, Austin Hospital, Melbourne, Australia.

${ }^{\mathrm{c}}$ Interventional Neuroradiology Unit, Monash Imaging, Monash Health, Melbourne, Australia.

${ }^{\mathrm{d}}$ Stroke Division, Florey Institute of Neuroscience and Mental Health, University of Melbourne, Australia and Department of Neurology, Austin Health, Melbourne, Australia.

${ }^{\mathrm{e}}$ Department of Imaging, Monash University, Melbourne, Australia.

${ }^{\mathrm{f}}$ School of Medicine, Faculty of Health, Deakin University, Pigdons Road, Waurn Ponds, VIC 3216, Australia.

${ }^{\text {g}}$ South Australian Health and Medical Research Institute, Adelaide, Australia.

This is the author manuscript accepted for publication and has undergone full peer review but has not been through the copyediting, typesetting, pagination and proofreading process, which may lead to differences between this version and the Version of Record. Please cite this article as doi: 10.1111/imj.14069

This article is protected by copyright. All rights reserved. 
${ }^{\mathrm{h}}$ The University of Adelaide, South Australia, Australia.

${ }^{\mathrm{i}}$ Neuroendovascular Program, Massachusetts General Hospital, Harvard Medical

School, Boston, Massachusetts, USA.

\section{Corresponding Author:}

Dr Julian Maingard

Department of Radiology, Austin Hospital, 145 Studley Road, Heidelberg, VIC 3084

E-mail address: julian.maingard@gmail.com

\section{Short Title:}

Thrombectomy outcomes with and without thrombolysis in acute stroke

\section{Keywords:}

Interventional neuroradiology, endovascular, stroke, large vessel occlusion, thrombectomy, thrombolysis, reperfusion, IV tPA

Manuscript Word Count: 1987 (excluding abstract, tables, legends and references)

Abstract word count: 243

\section{Introduction}

Endovascular thrombectomy (EVT) is now considered the standard of care in the management of acute ischaemic stroke (AIS) secondary to large vessel occlusion (LVO) in the anterior circulation. Evidence stems from large randomised controlled trials, including large pooled analyses demonstrating improved functional

This article is protected by copyright. All rights reserved. 
independence using combined EVT and bridging thrombolysis with intravenous (IV) tissue plasminogen activator (tPA) over thrombolysis alone ${ }^{1-8}$. While the benefit of thrombolysis within 4.5 hours of AIS has been firmly established ${ }^{9-13}$, studies suggest limitations in its ability to achieve acceptable reperfusion rates in LVO cases. ${ }^{14-18}$ Given these potential limitations, uncertainties remain as to the degree of benefit obtained with bridging thrombolysis when used in conjunction with EVT for LVO AIS or whether this practice confers additional risk.

The administration of thrombolysis not only presents a significant cost to health care systems ${ }^{19,20}$, but may impact on delays in time from imaging to groin puncture in comprehensive stroke centres, or time from imaging to patient transfer to these centres. $^{19,21-23}$ These potential delays are of concern given the time dependent effect on EVT outcomes. ${ }^{24}$ Thrombolysis is also associated with small but theoretical risks including anaphylaxis, early recurrent ischaemic stroke and coagulopathy, the latter potentially resulting in systemic or intracranial haemorrhage. ${ }^{19,22,25}$

Multiple single-centre studies have analysed AIS patient outcomes for EVT without thrombolysis, reporting comparable functional and safety outcomes when compared to EVT in addition to thrombolysis. ${ }^{20,22,23,26-28}$ However, a recent meta-analysis of the original randomised controlled trials showed conflicting evidence from these single-centre studies and reported lower rates of 90-day severe functional impairment or death as indicated by a modified Rankin Scale (mRS) of 5-6 and a trend towards 
improved 90-day functional outcome in patients receiving thrombolysis alongside EVT. ${ }^{29}$ The aim of this study was to investigate the clinical outcomes in AIS patients undergoing EVT with or without bridging thrombolysis with IV alteplase.

\section{Methods}

We performed a retrospective review of a prospectively collected database over a 6year period between June 2010-July 2016 from 24 hospitals in a large catchment area in the Republic of Ireland. A 'drip and ship' model of care was utilised with hospital distances to the primary stroke centre (with $24 / 7$ endovascular capability) ranging from 5.4 to 233 kilometres. Baseline patient demographics, clinical characteristics, imaging findings and clinical outcomes were analysed. Patients were eligible for inclusion provided all such details were available for analysis. Clinical outcome was assessed based on the mRS, with a favourable 90-day functional outcome defined as a score of 0-2 indicating functional independence. Successful reperfusion was defined as a modified Thrombolysis in Cerebral Infarction (mTICI) score of e2b. Symptomatic intracranial haemorrhage (sICH) was defined as intracranial haemorrhage associated with deterioration from baseline National Institutes of Health Stroke Scale (NIHSS) by e 4 points. Patients were divided into two groups based on either having received thrombolysis or proceeding to EVT directly (no thrombolysis). Some patients included in this retrospective review were also included in the interventional arm of the ESCAPE trial $(n=17) .^{3}$ 
Patients with AIS were selected for EVT using the same selection criteria as those utilised by the ESCAPE trial. ${ }^{3}$ Thus, all patients were evaluated with an initial unenhanced CT brain, allowing for assessment of the Alberta Stroke Program Early CT Score (ASPECTS). ${ }^{30}$ Next, multiphase CT angiography was performed and provides assessment of intracranial arterial occlusion(s) and grading of the leptomeningeal collateral circulation. All patients eligible for thrombolysis received IV alteplase, however failure to receive thrombolysis did not preclude patients from EVT. All patients received standard of care for a LVO AIS at their respective centres, and intervention with EVT was instigated based on eligibility criteria previously described $^{3}$. Patients with a contraindication for thrombolysis who were eligible for EVT received EVT alone.

Statistical analyses were performed with SPSS 23 (IBM, Armonk, NY). Continuous data were expressed as mean \pm standard deviation $(\mathrm{SD})$ for parametric means or median and interquartile range (IQR) otherwise. Odds ratios were expressed with a 95\% confidence interval. Continuous variables were analysed with one-way analysis of variance (ANOVA) or Mann-Whitney $U$ tests for parametric and nonparametric data, respectively. Categorical data was analysed with Pearson Chi-Square or Fisher's exact tests. Univariate and multivariate logistic regression analyses were used to identify predictors of clinical outcome that were significantly influenced by the administration of bridging thrombolysis and both raw and adjusted odds ratios were calculated. These variables included age, sex, hypertension, hyperlipidaemia, 
ischemic heart disease, diabetes, atrial fibrillation, previous stroke, NIHSS, ASPECTS, collateral grade, general anaesthesia during EVT, number of passes, successful reperfusion mTICI e $2 \mathrm{~b}, \mathrm{sICH}$, interval from symptom onset to groin puncture and time to reperfusion. Changes in functional outcome were analysed in XLSTAT (Addinsoft, NY) using the Cochran-Mantel-Haenszel test (CMHT). Statistical significance was defined as pd0.05.

\section{Results}

\section{Baseline characteristics}

A total of 355 patients who underwent EVT for AIS were included in the study. As this was a prospectively collected database no patients were excluded from analysis. The mean age was $67 \pm 14$ years and $55 \%$ of the cohort were male. Bridging thrombolysis was administered in 210 patients (59\%) prior to EVT and the remaining 145 patients (41\%), ineligible for thrombolysis either due to delayed presentation or other clinical contraindications, underwent EVT directly. A 'drip and ship' model of care was utilised in 164 patients (46\%). Both groups were comparable in terms of stroke severity with a mean NIHSS score of 17 at presentation as well as similar ASPECTS on unenhanced CT at presentation (Table 1). The vast majority of EVT procedures were performed using local anaesthesia or light sedoanalgaesia only $(97 \%)$.

\section{Outcomes}

This article is protected by copyright. All rights reserved. 
Successful reperfusion (mTICI e $2 \mathrm{~b})$ was achieved in $80 \%(\mathrm{n}=284)$ of patients with a trend towards greater success in the thrombolysis group (Table 2). The reperfusion rate was higher in the group receiving IV tPA (unadjusted OR 2.2, 95\% CI: 1.29-3.73, $\mathrm{p}=0.004$ ) although this effect was attenuated when all variables were considered (adjusted OR [AOR] 1.22, 95\% CI: 0.60-2.5, $\mathrm{p}=0.580$ ). Patients who received thrombolysis also had significantly faster times from symptom onset-to-groin puncture (257 versus 342 minutes, $\mathrm{p}<0.001)$ and onset-to-first reperfusion $(297$ versus 385 minutes, $\mathrm{p}<0.001)$. However, the time from groin puncture to first reperfusion did not differ significantly between groups. Fewer thrombectomy passes ( 2 versus 3, $\mathrm{p}=0.012$ ) were required to achieve successful reperfusion in the thrombolysis group.

Tables 1 and 2 summarise the clinical outcomes for patients who received bridging thrombolysis. There was significantly better functional independence at 90 -days (adjusted OR [AOR] 2.17, 95\% CI:1.06-4.44, $\mathrm{p}=0.033$ ) in the thrombolysis group. There was a lower 90-day mortality in the thrombolysis group after adjusting for confounding factors (AOR $0.79,95 \% \mathrm{CI}$ : 0.36-1.74, $\mathrm{p}=0.551$ ). There were no significant differences in terms of collateral grade, major complication or sICH between groups. Overall, the distribution in 90-day $\mathrm{mRS}$ scores showed better functional independence in the thrombolysis group ( $p=0.571$, Cochran-MantelHaenszel test, Figure 1). 
Multivariate regression analysis, adjusting for all baseline clinical and technical parameters including NIHSS and ASPECTS, demonstrated that successful reperfusion was the strongest predictor of favourable 90-day clinical outcome (AOR 10.4, 95\% $\mathrm{CI}: 3.6-29.7, \mathrm{p}<0.001)$. Other factors associated with a good functional outcome were younger age (AOR 0.93, 95\% CI:0.90-0.96, $\mathrm{p}<0.001$ ), lesser stroke severity (AOR 0.89, 95\% CI:0.84-0.96, $\mathrm{p}=0.001$ ) and fewer thrombectomy passes (AOR 0.85, 95\% CI:0.75-0.97, $\mathrm{p}=0.013$ ). The 90-day mortality was increased in older patients (AOR 1.04, 95\% CI:1.00-1.07, $\mathrm{p}=0.048$ ) and those who developed sICH (AOR 3.94, 95\% CI:1.16-13.5, $\mathrm{p}=0.028)$. Baseline stroke severity, ASPECTS and collateral circulation grades were not predictive of 90-day outcomes.

\section{Discussion}

This study has shown that patients with AIS who received bridging thrombolysis prior to EVT had significantly reduced mortality and improved 90-day functional independence without additional major complications or sICH.

Multiple single-centre studies have compared outcomes in patients undergoing EVT in addition to IV thrombolysis versus EVT alone, reporting comparable reperfusion rates, functional outcomes, safety parameters and mortality rates between both groups. $^{20,22,23,26-28}$ Broeg-Morvay et al. also performed multivariate matching analysis in their study, demonstrating lower rates of sICH and lower mortality in patients treated with EVT alone. ${ }^{22}$ Interestingly, results from these single-centre 
studies differ from a meta-analysis evaluating outcomes for EVT with bridging thrombolysis versus EVT alone. ${ }^{29}$ In accordance with the Preferred Reporting Items for Systematic Reviews and Meta-Analysis (PRISMA) guidelines, seven of the major EVT randomised controlled trials were deemed eligible for inclusion in this metaanalysis: MR CLEAN, ESCAPE, EXTEND-IA, SWIFT PRIME, REVASCAT, THRACE and PISTE. ${ }^{2-6,8,31}$ While the meta-analysis results displayed substantial heterogeneity, patients who received IV thrombolysis in addition to EVT had a significantly lower rate of severe disability or death (mRS 5-6) and a non-significant trend towards better 90-day functional independence (mRS 0-2), similar to this study. These findings suggest that EVT should be used in parallel with IV thrombolysis, where possible.

However, this meta-analysis acknowledged several limitations, including the low sample size in the EVT monotherapy group and that the trials were not designed to assess the effect of IV thrombolysis in conjunction with EVT. Failure to randomly assign patients to IV thrombolysis versus placebo introduces variance in baseline patient characteristics and in times to groin puncture, confounding results with selection bias. This failure to randomise the thrombolysis arm may also be considered a limitation in several single-centre studies including ours and those by Leker et al., Rai et al., Weber et al. and Wee et al., in which IV thrombolysis was administered to all presenting patients unless contraindicated..$^{20,26-28}$

A similar 2017 meta-analysis evaluated for variation in outcomes between groups 
receiving endovascular clot retrieval with IV thrombolysis and endovascular treatment alone. Following PRISMA guidelines 13 studies were included including data from 7 randomised controlled trials (MR CLEAN, ESCAPE, REVASCAT, SWIFT PRIME, EXTEND IA, SWIFT and STAR). Analysis revealed results in accordance with other meta-analyses; IV thrombolysis combined with EVT had significantly improved 90-day functional independence (mRS 0-2; OR 1.27, 95\% CI $1.05-1.55, \mathrm{p}=0.02$ ) and significantly lower rates of mortality compared to EVT alone. These findings further support that IV thrombolysis, where not contraindicated, should be used together with EVT. ${ }^{32}$

Other analyses have reached different conclusions. Subgroup analysis by the HERMES collaboration suggests benefit of EVT for patients presenting with LVO AIS, who were not treated with IV thrombolysis. ${ }^{7}$ In their pooled analysis of the SWIFT and STAR studies, Coutinho et al, did not find significant benefit in functional outcomes with bridging IV thrombolysis. However, interestingly, even after adjusting for confounders such as international normalised ratio (INR) and antiplatelet use, they found lower rates of sICH (adjusted OR 0.03, 95\% CI 0.00-1.28) in those receiving bridging IV tPA, ${ }^{33}$ IV thrombolysis can have significant implications on time to intervention, health care expenditure and patient safety. However, thrombolysed patients may benefit from treatment of distal emboli, a degree of reperfusion in cases of failed EVT and partial lysis of the thrombus 
reducing the number of stent-retriever passes and procedure times. ${ }^{19}$

Our results showed benefit of bridging thrombolysis prior to EVT for patients with AIS, with significantly reduced mortality and improved 90-day functional independence without additional major complications or sICH. There was a borderline trend towards better reperfusion rates in the thrombolysis group. These results can be partially explained through the effects of tPA on clot lysis in aiding cerebral reperfusion. It has been shown that tissue plasminogen factors play a role in the clotting cascade as fibrinolytics via the conversion of plasminogen into plasmin. Thus, it would seem that the use of IV thrombolysis before EVT acts to lyse the offending clot to some extent, if not completely. ${ }^{34} \mathrm{IV}$ thrombolysis may result in clot lysis prior to EVT, or partially lyse the clot to permit some reperfusion before EVT is conducted, resulting in reduced time of cerebral hypoxia and mitigating irreversible neuronal damage. Additionally, partial clot lysis may allow for more successful reperfusion with EVT as suggested by the need for fewer thrombectomy passes to achieve reperfusion in our cohort.

Our findings contrast with several published studies which suggest that IV thrombolysis can be problematic in stroke treatment, potentially resulting in higher rates of sICH and lower rates of successful reperfusion compared with cohorts treated with EVT alone. ${ }^{28,35}$ This may be attributed to the fact that many of these were smaller studies and were therefore underpowered to accurately evaluate these 
parameters. A larger study with higher power demonstrated an increase in sICH at 2436 hours with the use of thrombolysis and EVT, but showed no differences in functional independence or mortality at 90-days. ${ }^{36}$

The overall onset-to-groin puncture as well as onset-to-reperfusion times were longer in the EVT only (no thrombolysis) group of our cohort. This may be due to initial presentation outside the 4.5-hour time-window rendering patients in this group ineligible for thrombolysis. Nevertheless, there was no difference in groin puncture to first reperfusion time between groups. Successful reperfusion remained the strongest predictor of functional independence in our series.

There are several limitations to our study. Several satellite centres were involved in a 'drip and ship' model of care, making it difficult to account for variance in regional clinical practice and decisions on eligibility for IV thrombolysis. Being a retrospective study, the two treatment arms were not randomly allocated. Data was maintained on a central database at the time of presentation and was analysed retrospectively. Furthermore, unmeasured patient confounders such as anticoagulation, that contraindicate IV thrombolysis and indicate a population predisposed to poorer outcomes have the potential to impact results.

\section{Conclusion}

Our study demonstrates better 90-day functional independence in patients who 
received bridging IV thrombolysis prior to EVT versus direct EVT for the treatment of LVO AIS without additional risk of complications or sICH. These findings are similar to those reported previously in a meta-analysis and support the need for a prospective, randomised controlled trial to definitively address the role of IV thrombolysis in the era of EVT.

\section{Sources of Funding: None}

Disclosures: Dr. John Thornton is on the scientific advisory board for Neuravi, Ireland. Personal fees. Prof. Vincent Thijs has received consulting fees for Medtronic. Personal fees.

This article is protected by copyright. All rights reserved. 


\section{References}

1. Badhiwala JH, Nassiri F, Alhazzani W, et al. Endovascular Thrombectomy for Acute Ischemic Stroke: A Meta-analysis. Jama. 2015; 314: 1832-43.

2. Berkhemer OA, Fransen PS, Beumer D, et al. A randomized trial of intraarterial treatment for acute ischemic stroke. The New England journal of medicine. 2015; 372: 11-20.

3. Goyal M, Demchuk AM, Menon BK, et al. Randomized assessment of rapid endovascular treatment of ischemic stroke. The New England journal of medicine. 2015; 372: 1019-30.

4. Campbell BC, Mitchell PJ, Kleinig TJ, et al. Endovascular therapy for ischemic stroke with perfusion-imaging selection. The New England journal of medicine. 2015; 372: 1009-18.

5. Jovin TG, Chamorro A, Cobo E, et al. Thrombectomy within 8 hours after symptom onset in ischemic stroke. The New England journal of medicine. 2015; 372: 2296-306.

6. Saver JL, Goyal M, Bonafe A, et al. Stent-retriever thrombectomy after intravenous t-PA vs. t-PA alone in stroke. The New England journal of medicine. 2015; 372: 2285-95.

7. Goyal M, Menon BK, van Zwam WH, et al. Endovascular thrombectomy after large-vessel ischaemic stroke: a meta-analysis of individual patient data from five randomised trials. Lancet (London, England). 2016; 387: 1723-31.

8. Bracard S, Ducrocq X, Mas JL, et al. Mechanical thrombectomy after intravenous alteplase versus alteplase alone after stroke (THRACE): a randomised controlled trial. The Lancet Neurology. 2016; 15: 1138-47.

9. Tissue plasminogen activator for acute ischemic stroke. The National Institute of Neurological Disorders and Stroke rt-PA Stroke Study Group. The New England journal of medicine. 1995; 333: 1581-7.

10. Hacke W, Kaste M, Bluhmki E, et al. Thrombolysis with alteplase 3 to 4.5 hours after acute ischemic stroke. The New England journal of medicine. 2008; 359: 1317-29.

11. Lees KR, Bluhmki E, von Kummer R, et al. Time to treatment with intravenous alteplase and outcome in stroke: an updated pooled analysis of ECASS, ATLANTIS, NINDS, and EPITHET trials. Lancet (London, England). 2010; 375: 1695-703.

12. Wardlaw JM, Murray V, Berge E, et al. Recombinant tissue plasminogen activator for acute ischaemic stroke: an updated systematic review and meta-analysis. Lancet (London, England). 2012; 379: 2364-72.

13. Emberson J, Lees KR, Lyden P, et al. Effect of treatment delay, age, and stroke severity on the effects of intravenous thrombolysis with alteplase for acute ischaemic stroke: a meta-analysis of individual patient data from randomised trials. Lancet (London, England). 2014; 384: 1929-35.

14. Bhatia R, Hill MD, Shobha N, et al. Low rates of acute recanalization with intravenous recombinant tissue plasminogen activator in ischemic stroke: real-world experience and a call for action. Stroke; a journal of cerebral circulation. 2010; 41: 2254-8.

15. del Zoppo GJ, Poeck K, Pessin MS, et al. Recombinant tissue plasminogen activator in acute thrombotic and embolic stroke. Annals of neurology. 1992; 32: 78-86.

16. Mishra SM, Dykeman J, Sajobi TT, et al. Early reperfusion rates with IV tPA are determined by CTA clot characteristics. AJNR American journal of neuroradiology. 2014; 35: 2265-72.

17. De Silva DA, Brekenfeld C, Ebinger M, et al. The benefits of intravenous thrombolysis relate to the site of baseline arterial occlusion in the Echoplanar Imaging Thrombolytic Evaluation Trial (EPITHET). Stroke; a journal of cerebral circulation. 2010; 41: 295-9.

18. Rha JH and Saver JL. The impact of recanalization on ischemic stroke outcome: a metaanalysis. Stroke; a journal of cerebral circulation. 2007; 38: 967-73.

19. Chandra RV, Leslie-Mazwi TM, Mehta BP, et al. Does the use of IV tPA in the current era of rapid and predictable recanalization by mechanical embolectomy represent good value? Journal of neurointerventional surgery. 2016; 8: 443-6. 
20. Rai AT, Boo S, Buseman C, et al. Intravenous thrombolysis before endovascular therapy for large vessel strokes can lead to significantly higher hospital costs without improving outcomes. Journal of neurointerventional surgery. 2017.

21. Menon BK, Sajobi TT, Zhang Y, et al. Analysis of Workflow and Time to Treatment on Thrombectomy Outcome in the Endovascular Treatment for Small Core and Proximal Occlusion Ischemic Stroke (ESCAPE) Randomized, Controlled Trial. Circulation. 2016; 133: 2279-86.

22. Broeg-Morvay A, Mordasini P, Bernasconi C, et al. Direct Mechanical Intervention Versus Combined Intravenous and Mechanical Intervention in Large Artery Anterior Circulation Stroke: A Matched-Pairs Analysis. Stroke; a journal of cerebral circulation. 2016; 47: 1037-44.

23. Kass-Hout T, Kass-Hout O, Mokin M, et al. Is bridging with intravenous thrombolysis of any benefit in endovascular therapy for acute ischemic stroke? World neurosurgery. 2014; 82: e453-8.

24. Saver JL, Goyal M, van der Lugt A, et al. Time to Treatment With Endovascular

Thrombectomy and Outcomes From Ischemic Stroke: A Meta-analysis. Jama. 2016; 316: 1279-88.

25. Awadh M, MacDougall N, Santosh C, Teasdale E, Baird T and Muir KW. Early recurrent ischemic stroke complicating intravenous thrombolysis for stroke: incidence and association with atrial fibrillation. Stroke; a journal of cerebral circulation. 2010; 41: 1990-5.

26. Leker RR, Pikis S, Gomori JM and Cohen JE. Is Bridging Necessary? A Pilot Study of Bridging versus Primary Stentriever-Based Endovascular Reperfusion in Large Anterior Circulation Strokes. Journal of stroke and cerebrovascular diseases : the official journal of National Stroke Association. 2015; 24: 1163-7.

27. Weber R, Nordmeyer H, Hadisurya J, et al. Comparison of outcome and interventional complication rate in patients with acute stroke treated with mechanical thrombectomy with and without bridging thrombolysis. Journal of neurointerventional surgery. 2017; 9: 229-33.

28. Wee CK, McAuliffe W, Phatouros CC, et al. Outcomes of Endovascular Thrombectomy with and without Thrombolysis for Acute Large Artery Ischaemic Stroke at a Tertiary Stroke Centre.

Cerebrovascular diseases extra. 2017; 7: 95-102.

29. Tsivgoulis G, Katsanos AH, Mavridis D, et al. Endovascular thrombectomy with or without systemic thrombolysis? Therapeutic advances in neurological disorders. 2017; 10: 151-60.

30. Barber PA, Demchuk AM, Zhang J and Buchan AM. Validity and reliability of a quantitative computed tomography score in predicting outcome of hyperacute stroke before thrombolytic therapy.

ASPECTS Study Group. Alberta Stroke Programme Early CT Score. Lancet (London, England). 2000; 355: 1670-4.

31. Muir KW, Ford GA, Messow CM, et al. Endovascular therapy for acute ischaemic stroke: the Pragmatic Ischaemic Stroke Thrombectomy Evaluation (PISTE) randomised, controlled trial. Journal of neurology, neurosurgery, and psychiatry. 2017; 88: 38-44.

32. Mistry EA, Mistry AM, Nakawah MO, et al. Mechanical Thrombectomy Outcomes With and Without Intravenous Thrombolysis in Stroke Patients: A Meta-Analysis. Stroke; a journal of cerebral circulation. 2017; 48: 2450-6.

33. Coutinho JM, Liebeskind DS, Slater LA, et al. Combined Intravenous Thrombolysis and Thrombectomy vs Thrombectomy Alone for Acute Ischemic Stroke: A Pooled Analysis of the SWIFT and STAR Studies. JAMA Neurol. 2017; 74: 268-74.

34. Barreto AD. Intravenous thrombolytics for ischemic stroke. Neurotherapeutics : the journal of the American Society for Experimental NeuroTherapeutics. 2011; 8: 388-99.

35. Mao YT, Mitchell P, Churilov L, Dowling R, Dong Q and Yan B. Early Recanalization Postintravenous Thrombolysis in Ischemic Stroke with Large Vessel Occlusion: A Digital Subtraction Angiography Study. CNS neuroscience \& therapeutics. 2016; 22: 643-7.

36. Abilleira S, Ribera A, Cardona P, et al. Outcomes After Direct Thrombectomy or Combined Intravenous and Endovascular Treatment Are Not Different. Stroke; a journal of cerebral circulation. 2017; 48: 375-8. 


\begin{abstract}
Introduction

Endovascular thrombectomy (EVT) for management of large vessel occlusion (LVO) acute ischaemic stroke (AIS) is now current best practice. The aim of this study was to determine if bridging intravenous alteplase therapy confers any clinical benefit.
\end{abstract}

\title{
Methods
}

A retrospective study of patients treated with EVT for LVO was performed. Outcomes were compared between patients receiving thrombolysis and EVT with EVT alone. Primary endpoints were reperfusion rate, 90-day functional outcome and mortality using the modified Rankin scale (mRS) and symptomatic intracranial haemorrhage (sICH).

\section{Results}

A total of 355 patients who underwent EVT were included: 210 with thrombolysis (59\%) and 145 without (41\%). The reperfusion rate was higher in the group receiving IV tPA (unadjusted OR 2.2, 95\% CI: 1.29-3.73, $\mathrm{p}=0.004$ ) although this effect was attenuated when all variables were considered (adjusted OR [AOR] 1.22, 95\% CI: 0.60-2.5, $\mathrm{p}=0.580$ ). The percentage achieving functional independence (mRS 0-2) at 90-days was higher in patients who received bridging IV tPA (AOR 2.17, 95\% CI:1.06-4.44, $\mathrm{p}=0.033$ ). There was no significant difference in major complications including sICH (AOR 1.4, 95\% CI: 0.51-3.83, $\mathrm{p}=0.512$ ). There was lower 90 -day mortality in the bridging IV tPA group (AOR 0.79, 95\% CI: $0.36-1.74, \mathrm{p}=0.551)$. 
Fewer thrombectomy passes ( 2 versus $3, \mathrm{p}=0.012$ ) were required to achieve successful reperfusion in the IV tPA group. Successful reperfusion (mTICI e2b) was the strongest predictor for 90-day functional independence (AOR 10.4, 95\% CI:3.6-29.7, p<0.001).

\section{Conclusions}

Our study supports the current practice of administering intravenous alteplase before endovascular therapy. 
Table 1. Baseline clinical, imaging and outcome characteristics of both study groups.

\begin{tabular}{|c|c|c|c|}
\hline Outcome variable & $\begin{array}{l}\text { Thrombolysis } \\
\text { (n=210) }\end{array}$ & $\begin{array}{l}\text { No thrombolysis } \\
\qquad(\mathrm{n}=145)\end{array}$ & p-value \\
\hline Mean age, years (SD) & $66(14)$ & $68(14)$ & 0.271 \\
\hline Male sex, n (\%) & $116(55)$ & $81(56)$ & 0.914 \\
\hline Median ASPECTS (IQR) & $9(2)$ & $9(3)$ & $0.029 *$ \\
\hline Mean NIHSS score (SD) & $17(5)$ & $17(6)$ & 0.812 \\
\hline Grade 2 or 3 collateral circulation, $\mathrm{n}(\%)$ & $93(55)$ & $79(66)$ & 0.065 \\
\hline $\begin{array}{l}\text { Mean onset time to groin puncture, mins } \\
\text { (SD) }\end{array}$ & $257(104)$ & $342(223)$ & $<0.001^{*}$ \\
\hline $\begin{array}{l}\text { Mean onset time to first reperfusion, } \\
\text { mins (SD) }\end{array}$ & $297(110)$ & $385(225)$ & $<0.001^{*}$ \\
\hline $\begin{array}{l}\text { Median time from groin puncture to first } \\
\text { reperfusion, mins (IQR) }\end{array}$ & $32(30)$ & $33(38)$ & 0.330 \\
\hline Successful reperfusion $\dagger, \mathrm{n}(\%)$ & $179(85)$ & $105(72)$ & $0.003 *$ \\
\hline Median number of EVT passes, $n$ (IQR) & $2(3)$ & $3(5)$ & $0.012 *$ \\
\hline Major complication, $\mathrm{n}(\%)$ & $24(11)$ & $21(15)$ & 0.395 \\
\hline Symptomatic ICH, n (\%) & $15(7)$ & $14(10)$ & 0.396 \\
\hline $\begin{array}{l}\text { Functional independence (mRS 0-2) at } 3 \\
\text { months, } \mathrm{n}(\%)\end{array}$ & $113(54)$ & $49(34)$ & $<0.001 *$ \\
\hline Mortality at 3 months, $\mathrm{n}(\%)$ & $33(16)$ & $45(31)$ & $0.001 *$ \\
\hline
\end{tabular}

$\mathrm{n}=$ Number, $\mathrm{IQR}=$ Interquartile range, $\mathrm{SD}=$ Standard deviation, $\mathrm{ICH}=$ Intracranial haemorrhage, $\mathrm{mRS}=$ modified Rankin score, $\dot{\dagger}=\mathrm{TICI} 2 \mathrm{~b}$ or greater. *indicates statistical significance. 
Table 2. Univariate and multivariate logistic regression outcomes for patients who received bridging intravenous thrombolysis prior to thrombectomy.

\begin{tabular}{|l|c|c|c|c|}
\hline Outcome variable & $\begin{array}{c}\text { Unadjusted OR } \\
(\mathbf{9 5 \%} \text { CI) }\end{array}$ & p-value & Adjusted OR & p-value \\
\hline Functional independence (mRS & $2.28(1.47-3.54)$ & $<0.001^{*}$ & $2.17(1.06-4.44)$ & $0.033^{*}$ \\
0-2) at 3 months & & & & \\
\hline Mortality at 3 months & $0.41(0.25-0.69)$ & $0.001^{*}$ & $0.79(0.36-1.74)$ & 0.551 \\
\hline Successful reperfusion† & $2.20(1.29-3.73)$ & $0.004^{*}$ & $1.22(0.60-2.50)$ & 0.580 \\
\hline Good collateral grade (2-3) & $0.64(0.39-1.03)$ & 0.069 & $0.72(0.42-1.23)$ & 0.225 \\
\hline Major complication & $0.76(0.41-1.43)$ & 0.420 & $1.15(0.52-2.57)$ & 0.730 \\
\hline Symptomatic ICH & $0.72(0.34-1.54)$ & 0.434 & $1.40(0.51-3.83)$ & 0.512 \\
\hline
\end{tabular}

$\mathrm{n}=$ Number, $\mathrm{OR}=$ Odds Ratio, $\mathrm{CI}=$ Confidence Interval, $\mathrm{ICH}=$ Intracranial haemorrhage $(\mathrm{ICH}), \mathrm{mRS}=$ modified Rankin score, $\uparrow=\mathrm{TICI} 2 \mathrm{~b}$ or greater. *indicates statistical significance. 


\title{
OUTCOMES OF ENDOVASCULAR THROMBECTOMY WITH AND WITHOUT BRIDGING THROMBOLYSIS FOR ACUTE LARGE VESSEL OCCLUSION ISCHAEMIC STROKE
}

\begin{abstract}
Authors and Affiliations:
Julian Maingard ${ }^{\mathrm{b}, \mathrm{f}}$, Yasmin Shvarts ${ }^{\mathrm{b}}$, Ronan Motyer $^{\mathrm{a}}$, Vincent Thijs ${ }^{\mathrm{d}}$, Paul Brennan ${ }^{\mathrm{a}}$, Alan O’Hare $^{\mathrm{a}}$, Seamus Looby ${ }^{\mathrm{a}}$, John Thornton ${ }^{\mathrm{a}}$, Joshua A Hirsch ${ }^{\mathrm{i}}$, Christen D Barras ${ }^{\mathrm{g}, \mathrm{h}}$, Ronil V Chandra $^{\text {c,e }}$, Mark Brooks ${ }^{\text {b,d,f }}$, Hamed Asadi ${ }^{\text {a,b,c,e,f }}$, Hong Kuan Kok ${ }^{a}$
\end{abstract}

${ }^{a}$ Interventional Neuroradiology Service, Department of Radiology, Beaumont Hospital, Dublin 9, Ireland.

${ }^{\mathrm{b}}$ Interventional Neuroradiology Service, Radiology Department, Austin Hospital, Melbourne, Australia.

${ }^{\mathrm{c} I n t e r v e n t i o n a l ~ N e u r o r a d i o l o g y}$ Unit, Monash Imaging, Monash Health, Melbourne, Australia.

${ }^{\mathrm{d}}$ Stroke Division, Florey Institute of Neuroscience and Mental Health, University of Melbourne, Australia and Department of Neurology, Austin Health, Melbourne, Australia.

${ }^{\mathrm{e}}$ Department of Imaging, Monash University, Melbourne, Australia.

${ }^{\mathrm{f}}$ School of Medicine, Faculty of Health, Deakin University, Pigdons Road, Waurn Ponds, VIC 3216, Australia.

${ }^{\text {g}}$ South Australian Health and Medical Research Institute, Adelaide, Australia.

${ }^{\mathrm{h}}$ The University of Adelaide, South Australia, Australia.

${ }^{\mathrm{i}}$ Neuroendovascular Program, Massachusetts General Hospital, Harvard Medical School, Boston, Massachusetts, USA.

\section{Corresponding Author:}


Dr Julian Maingard

Department of Radiology, Austin Hospital, 145 Studley Road, Heidelberg, VIC 3084

E-mail address: julian.maingard@gmail.com

\section{Short Title:}

Thrombectomy outcomes with and without thrombolysis in acute stroke

\section{Keywords:}

Interventional neuroradiology, endovascular, stroke, large vessel occlusion, thrombectomy, thrombolysis, reperfusion, IV tPA

Manuscript Word Count: 1987 (excluding abstract, tables, legends and references)

Abstract word count: 243

This article is protected by copyright. All rights reserved. 


\section{University Library}

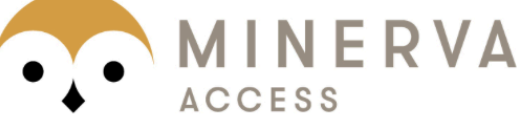

A gateway to Melbourne's research publications

Minerva Access is the Institutional Repository of The University of Melbourne

Author/s:

Maingard, J;Shvarts, Y;Motyer, R;Thijs, V;Brennan, P;O'Hare, A;Looby, S;Thornton, J;Hirsch, JA;Barras, CD;Chandra, R;Brooks, M;Asadi, H;Kok, HK

Title:

Outcomes of endovascular thrombectomy with and without bridging thrombolysis for acute large vessel occlusion ischaemic stroke

Date:

2019-03-01

Citation:

Maingard, J., Shvarts, Y., Motyer, R., Thijs, V., Brennan, P., O'Hare, A., Looby, S., Thornton, J., Hirsch, J. A., Barras, C. D., Chandra, R., Brooks, M., Asadi, H. \& Kok, H. K. (2019).

Outcomes of endovascular thrombectomy with and without bridging thrombolysis for acute large vessel occlusion ischaemic stroke. INTERNAL MEDICINE JOURNAL, 49 (3), pp.345-+. https://doi.org/10.1111/imj.14069.

Persistent Link:

http://hdl.handle.net/11343/285533 